\title{
Gateway Architecture for DVB-RCS Satellite Networks
}

\author{
Antonio Pietrabissa ${ }^{1}$ and Cristiana Santececca ${ }^{2}$
}

University of Rome "La Sapienza"

Dipartimento di Informatica e Sistemistica (DIS)

pietrabissa@dis.uniroma1.it, 2cristiana.santececca@tin.it

\begin{abstract}
The introduced gateway architecture for DVB-based geostationary satellite networks has been developed for the European Community project "GOECAST" (multiCAST over GEOstationary satellites) and aims at supporting the real-time (RT) traffic feeding the Gateway Earth Stations (GES). GESs provide the access to the satellite network to a large number of RT flows, whose delay constraints have to be guaranteed without affecting the lower priority traffic. A tight control on the queuing delays is obtained by the proposed scheduling scheme, while the proposed buffering scheme allows the scalability requirement to be met by means of a proper traffic aggregation criterion. Finally, the 'stolen slot' concept is introduced: whenever the RT traffic transmission rate is lower than the nominal rate, the 'stolen-slot' procedure allows the utilisation of the leftover bandwidth, considering also the multicast issues. Simulations have been performed with the OPNET tool to test the effectiveness of the proposed schemes and algorithms.
\end{abstract}

\section{The GEOCAST Project}

The GEOCAST scenario consists of a geostationary (GEO) satellite network with an on-board packet-switch, a Network Control Centre (NCC), in charge of several key tasks relevant to resource management and connection handling, several User Earth Stations (UES), which provide the access to few User Terminals (UT), and a limited number of Gateway Earth Stations (GES), which provide the access to a large population of users and to backbone networks. The GES uplink access is TDM (Time Division Mutiplexing): the uplink capacity is divided into time-slots; each time-slot is capable of transporting one packet. The UES uplink, conversely, is MF-TDMA (Multi Frequency Time-Division Multiple Access), so that the UESs are capable of sharing the uplink capacity. Fig. 1 shows the GEOCAST scenario.

While each GES has the exclusive use of the TDM uplink frames, the downlink capacity is shared among several GESs and UESs. Thus, the NCC, which manages the network resources, grants the requested rates of the GES connections; in particular, the Connection Admission Control (CAC) grants the requested capacity after the connection set-up for the connection life-time, while the Bandwidth-on-Demand (BoD) scheme (presented in [1]) grants some capacity in response to the capacity requests.

The protocol stack of the GEOCAST project is compliant with the DVB-RCS (Return Channel via Satellite-Digital Video Broadcasting) standard [2], [3] . In particu- 
lar, it uses the DVB-RCS ATM (Asynchronous Transfer Mode) format on the uplink and the DVB-S MPEG2 format on the downlink [4]]. Without explaining the overall protocol stack in detail, this means that the IP traffic entering the Gateway is mapped onto ATM connections, which are transported through the DVB priority traffic classes ([3]): real-time (RT), jitter tolerant (JT) and best effort (BE).

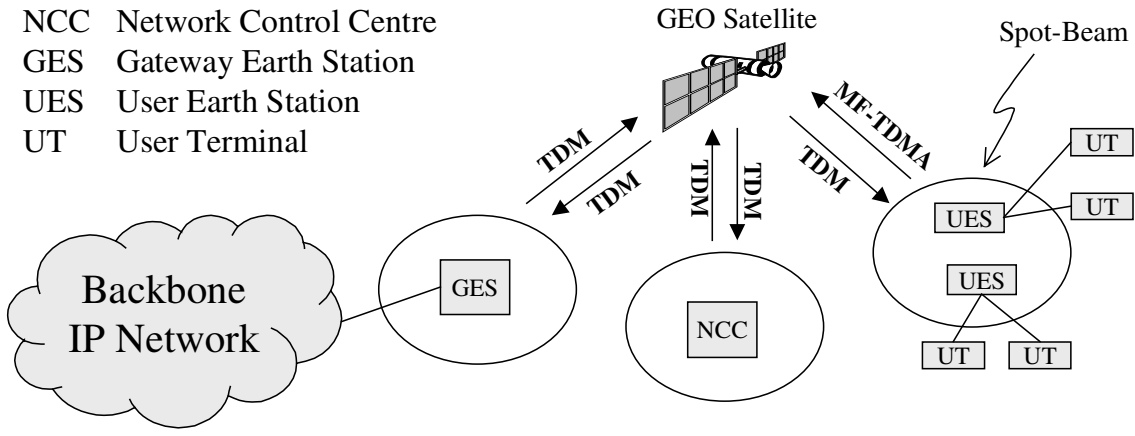

Fig. 1. GEOCAST scenario

This paper deals with the RT priority traffic, which is used to transport delay sensitive ATM connections; the ATM connections are characterised by the declared Quality of Service (QoS) parameters, as the Cell Transfer Delay (CTD) and the Cell Delay Variation (CDV), and by the declared traffic parameters, as the Peak Cell Rate (PCR). The CTD is the maximum acceptable delay perceived by the ATM cells from the source GES to the destination GES or UES; the CDV is the maximum allowed difference among the delays perceived by the ATM cells of the connection (jitter); the PCR is the maximum transmission rate. The ATM connections feeding the GES are subject to traffic shaping and policing through the Usage Parameter Control (UPC) blocks [5].

Furthermore, the GEOCAST network supports multicast traffic, which is mapped onto ATM one-to-many connections. For instance, a multicast session involving $n$ users is mapped onto $n$ one-to-many connections, each one with one sender and $(n-1)$ receivers. The GEOCAST on-board switch is capable of duplicating the received packets and of forwarding them towards the proper downlinks.

In Section 2, the problems related to the satellite networks are highlighted; in Section 3, a Gateway architecture is proposed; in Section 4, the 'stolen slot' concept is defined taking into account the multicast issues; Section 5 presents the simulations results; finally, in Section 6 the conclusions are drawn.

\section{Real-Time (RT) Traffic over Satellite Networks}

The uplink TDM frame of the GESs is divided into frames. Each frame consists of a number $N$ of time-slots and its duration is equal to $T_{F R A M E}$. Each time-slot is capable of transporting one ATM cell (hereinafter referred to as packet). In the GEOCAST project, $N=1136, T_{\text {FRAME }}=53 \mathrm{~ms}$ and each time-slot has a capacity $C_{S L O T}=8 \mathrm{kbps}$ (i.e., the capacity equivalent to 1 time-slot per frame is $8 \mathrm{kbps}$ ). 
The RT traffic has tight delay constraints, thus it cannot avail of the BoD mechanism, since the time required by the NCC to fulfil the bandwidth requests is equal to the Round Trip Delay (RTD) of the geostationary networks, which is about $500 \mathrm{~ms}$ and is likely to be greater than the CTD of the RT connections. As a consequence, the network cannot react to the rate variations and, therefore, during the connection set-up, the CAC in the NCC must reserve a capacity equal to the Peak Cell Rate (PCR) for the connection lifetime. In the one-to-many connection case, the PCR capacity must be reserved onto each involved downlink. Finally, the GES must check whether the required PCR is less than its available capacity, i.e., the uplink capacity minus the capacity already reserved for other active connections.

Usually [6].[9], the RT connections are associated to one or more logical channels, that is one or more time-slot per frame are assigned to the connection.

This solution has a problem of over-allocation, since where the number of timeslots $\mathrm{N}_{\mathrm{PCR}}$ required to map the connection is given by the following equation: $N_{P C R}=\lceil$ $P C R / C_{S L O T}$ T. On average, each connection needs $0.5 C_{S L O T}=4 \mathrm{kbps}$ more than its PCR. In addition, this scheme requires that each ATM connection - named Virtual Channel Connection (VCC) - has its own buffer in the GES. This buffering scheme causes a scalability problem for the GESs, because of the large number of connections the GESs have to support. Furthermore, the queues in the buffers, and thus the CDV of the connections, depend on the allocation policy and on the declared PCR.

As a mater of fact, if the channels are contiguous, the minimum queuing delay is zero, if the packet arrives in the buffer when the time-slot is available, while the maximum queuing delay is about $T_{F R A M E}$, if the packet arrives in the buffer just after the reserved time-slot. Thus, in this case, the CDV is about $T_{\text {FRAME. }}$ Fig. 2 a) shows an example of this mapping policy. Note that the arrival time $t_{a}(j)$ of the $j^{\text {th }}$ packet is equal to the beginning time of the time-slot used to transmit the packet itself; thus, the queuing delay perceived by the $j^{\text {th }}$ packet is zero. On the other hand, the arrival time $t_{a}(j+1)$ of the $(j+1)^{t h}$ packet is slightly greater than the beginning time of the assigned time-slot; thus, the packet can be transmitted only in the beginning of the following frame. Thus, the queuing delay perceived by the $(j+1)^{\text {th }}$ packet is about $T_{\text {FRAME }}$.

If the GES allocates the time-slots regularly over the frame, it manages to limit the CDV to $T_{\text {FRAME }} / N_{P C R}$. Fig. 2 b) shows an example of this mapping policy, in which the PCR of the connection is such that $N_{P C R}=3$ time-slots per frame. Note that the arrival time $t_{a}(j)$ of the $j^{\text {th }}$ packet is slightly greater than the beginning time of the assigned time-slot; thus, the packet can be transmitted only via the successive time-slot. Thus, the queuing delay perceived by the $j^{\text {th }}$ packet is about $T_{\text {FRAME }} / N_{\text {SLOT }}$. On the other hand, the arrival time $t_{a}(j+1)$ of the $(j+1)^{t h}$ packet is equal to the beginning time of the timeslot used to transmit the packet itself; thus, the queuing delay perceived by the $(j+1)^{t h}$ packet is zero. Note, however, that a regular allocation might be impossible because of already allocated time-slots; in this case, the connection suffers from a CDV $>T_{\text {FRAME }}$ / $N_{P C R}$.

In conclusion, the drawbacks of this architecture are: i) over-allocation of uplink resources; ii) scalability due to the per-VCC buffering scheme; iii) obtainable CDV limited by $T_{\text {FRAME }}$ or PCR iv) possible connection denial due to the impossibility of 
mapping the connection properly. In the following Section, the proposed architecture is presented, which overcomes the above mentioned problems.

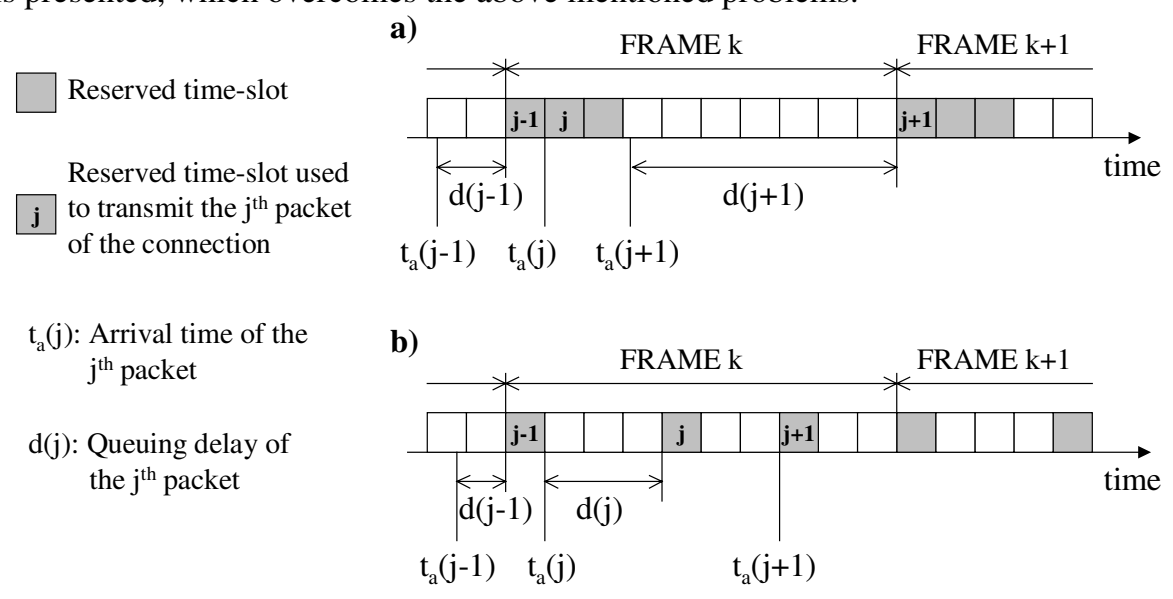

Fig. 2. Queuing delays associated to the frame mapping policies

\section{Proposed Gateway Architecture}

The proposed GES architecture aims at allowing the transmission of the RT connections to be independent of the uplink frame structure and at increasing the scalability.

This latter issue is addressed through an appropriate traffic aggregation policy. First of all, the RT VCC of the GES directed towards the same downlink (or downlinks in the case of the one-to-many connections) are aggregated into Virtual Path Connections (VPC); consequently, the VPC are defined by the couple \{Source GES, Destination Downlink(s) \}. Then, within each VPC, the VCCs are aggregated on the basis of their QoS, so that the VCCs characterized by similar CDV requirements are collected in the same buffer. This aggregation policy allows a great reduction of the buffer number, and, as a consequence, of the complexity of the scheduling algorithm (as it will be explained later), without affecting the QoS perceived by the distinct VCCs. As a matter of fact, in order to assure a certain CDV the GES must limit the queuing delay; if the queuing delay of the QoS buffer is kept under the most stringent CDV requirements among the ones of its VCC, the CDV target is met by each VCC.

In order to render the transmission of the RT packets independent of the frame structure of the uplink, the following considerations are taken into account:

- The transmission rates of the RT connections feeding the GES are controlled by the UPC blocks, so that the packet rate of each connection cannot exceed the PCR.

- The RT connections are subject to the CAC, which assure that an uplink capacity equal to the PCR is always available.

Thus, the RT packets feeding the QoS buffers have always the matching available capacity on the uplink, and, because of the greater priority of the RT traffic with respect to the non real-time (NRT) traffic, should be transmitted as soon as possible. 
Therefore, the proposed architecture features a two-levels scheduling scheme (see Fig. 3):

1. The first scheduling level consists of a strict priority scheduler, which decides whether a RT packet or a NRT packet must be transmitted; this scheduler transmits NRT packets only if the RT buffer queues are empty.

2. The second scheduling level consists of a RT and a NRT schedulers, which decides which packets to send among the ones waiting in the RT and NRT queues, respectively.

This paper is focused onto the RT traffic, thus only the RT scheduler will be examined. The Earliest Deadline First (EDF) scheduler seems to be adequate, because of the following reasons:

- The queuing delay is the only criterion, on the basis of which the RT packets must be scheduled;

- The maximum queuing delay allowed to a certain buffer $k, d_{M A X}(k)$, is known, since it given by the minimum CDV requirement of the VCCs aggregated in the buffer $k$;

- The EDF is known to to be optimum in the sense that it minimizes the total probability of exceeding the deadlines for all streams ([10], [11]).

The EDF scheduler computes the deadlines of the packets by adding $d_{\text {MAX }}(k)$ to the arrival time of the packets feeding the buffer $k$, and then it schedules the packet with the earliest deadline.

Thanks to the double aggregation of the VCCs in QoS classes and in VPCs, a twolevels scheduler can be implemented: the first EDF scheduler decides which packet to sent among the ones in the head of the QoS queues of the single VPCs, and has complexity $O\left(\log N_{Q o S}\right)$, where $N_{Q o S}$ is the number of QoS classes defined within the RT class; the second scheduler decides decides which packet to sent among the ones selected by the first level schedulers, and has complexity $O\left(\log N_{V P C}\right)$, where $N_{V P C}$ is the number of VPCs.

Fig. 3 shows the proposed GES architecture.

While the proposed architecture succeeds in enhancing the scalability and in overcoming the problems related to the connection mapping, it has a drawback. During the $j^{\text {th }}$ frame, the NRT scheduler is allowed to transmit packets towards the $k^{\text {th }}$ downlink up to a certain capacity $C_{N R T}(j, k)$, which is granted by the NCC (by the CAC and by the BoD protocol). Since it is not likely that the RT sources transmit continuously at the $\mathrm{PCR}$, the capacity available to the RT traffic on the $k^{\text {th }}$ downlink which is left unused should increase $C_{N R T}(j, k)$. In the reference architecture, the RT connections are explicitly mapped onto the uplink frame; thus, if the RT connection buffer is empty in the beginning of an assigned time-slot, it can straightforwardly used by the NRT traffic. This simple procedure cannot be followed in the proposed architecture case. In the following Section, a procedure suitable for the proposed architecture is presented. 


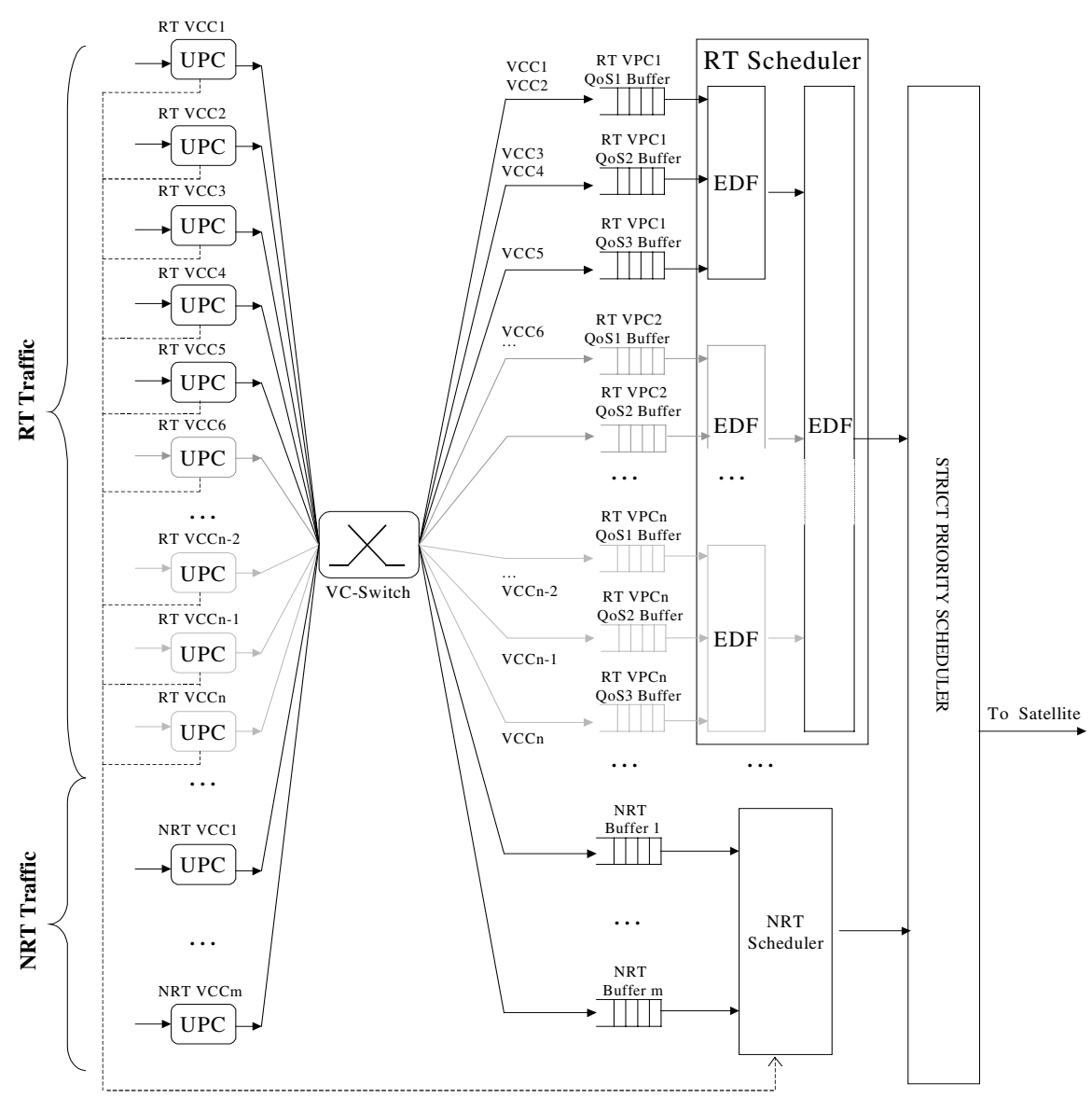

Fig. 3. Proposed gateway architecture

\section{4 'Stolen-Slot' Procedure for Unicast and Multicast Traffic}

Every time the RT connections transmit with a lower rate than the declared one, some capacity is left unused; when this capacity is equal to the time-slot capacity $\mathrm{C}_{\mathrm{SLOT}}$, one NRT packet can be served, and the time-slot is considered as 'stolen'. Two problems arise: i) how to compute the 'stolen' capacity, ii) how to assign the 'stolen' time-slots.

In order to identify whether a time-slot can be 'stolen', the proposed algorithm makes use of the UPC blocks of the GESs [5]). Each VCC is characterised by the peak transmission rate PCR. When the first packet of the VCC arrives at $t=t_{a}(1)$, the associated UPC block computes the Theoretical Arrival Time (TAT) of the second packet: $T A T_{2}=T A T_{1}+1 / P C R$, where $T A T_{1}=0$. Then, when the second packet arrives at $t=t_{a}(2)$, the UPC block performs the following actions (neglecting the policing function): i) if $t_{a}(2) \geq T A T_{2}, T A T_{3}=t_{a}(2)+1 / P C R$; ii) if $t_{a}(2) \leq T A T_{2}, T A T_{3}=T A T_{2}+$ 
1/PCR. Similarly, on the basis of $T A T_{k}$, when the $k^{\text {th }}$ packet arrives at $t_{a}(k)$ the UPC computes $T A T_{(k+1)}$.

The 'stolen-slots' algorithm is based on the fact that when a cell arrives at time $t_{a}(k)$ after its $T A T(k)$, then the next $T A T(k+1)$ is delayed. In particular, $T A T(k+1)$ is delayed by $\left[t_{a}(k)-T A T(k)\right]$. Since the TATs cannot be anticipated, this time is 'stolen'. When the sum of the stolen times is equal to $1 / P C R$, then 1 time-slot can be 'stolen', and the UPC block communicates it to the NRT scheduler (see Fig. 3). Fig. 4 shows an example run of the algorithm..

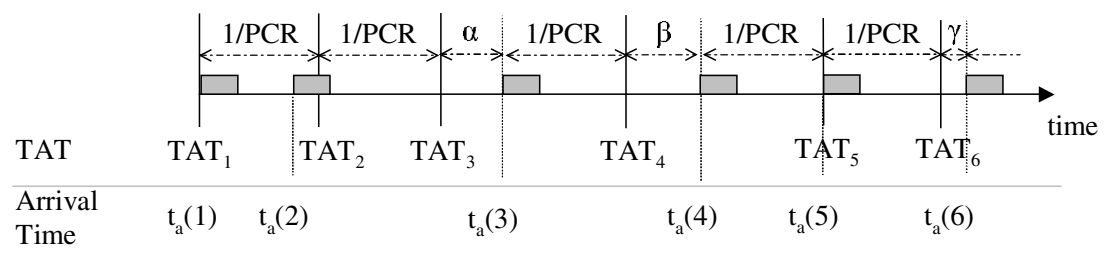

\begin{tabular}{lcccccc}
\hline $\begin{array}{l}\text { Stolen } \\
\text { Time }\end{array}$ & 0 & 0 & $\alpha=0.5 / \mathrm{PCR}$ & $\beta=0.6 / \mathrm{PCR}$ & 0 & $\gamma=0.2 / \mathrm{PCR}$ \\
$\begin{array}{l}\text { Tot. Stolen } \\
\text { Time }\end{array}$ & 0 & 0 & $0.5 / \mathrm{PCR}$ & $1.1 / \mathrm{PCR}$ & $0.1 / \mathrm{PCR}$ & $0.3 / \mathrm{PCR}$ \\
$\begin{array}{l}\text { Stolen } \\
\text { Slots }\end{array}$ & 0 & 0 & 0 & 1 & 1 & 1
\end{tabular}

Fig. 4. Example of the stolen slot algorithm

After that a time-slot is stolen, it has to be assigned to the proper connection. We recall that, while each GES has the exclusive use of the TDM uplink frames, the downlink capacity is shared among several GESs and UESs by the NCC. Thus, in the beginning of each frame, the GES is allowed to transmit a known capacity towards each downlink. In the case of one-to-many connections, the capacity is reserved by the NCC on each downlink involved by the connection, as shown in Fig. 5, in which the one-to-many VPC0 has one packets to send during the $j^{\text {th }}$ frame and is allowed to transmit two packets (i.e., it has two allocated time-slots).
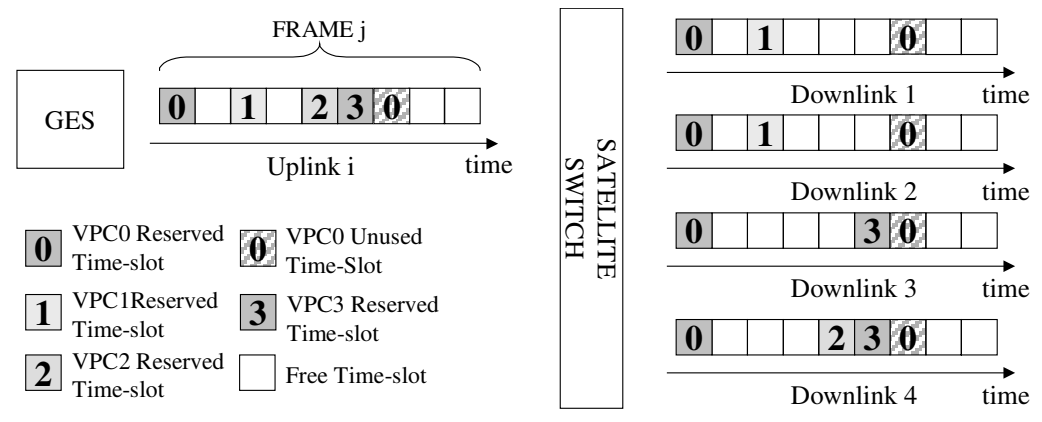

Fig. 5. Uplink and downlink reserved capacity

Let $\Delta$ be the set of the downlinks involved by the VPC the stolen slot belongs to. The problem is to decide which VPC should steal the time-slot: 
1. First of all, the GES must check which are the eligible VPCs on the basis of the following rules: i) the VPC buffer in the GES must not be empty; ii) the set $D(j)$ of the downlinks involved by the VPCj is a subset of $\Delta: D(j) \subseteq \Delta$.

2. Then, the eligible VPCs are ordered with respect to i) the priority traffic and ii) the number of involved downlinks, i.e., the cardinality of $D(j)$.

3. Finally, the time-slot is given to the first VPC of the list.

Note that, in the one-to-many case, the time-slot might be stolen by a certain VPCj, which does not involve all the downlinks, on which the capacity has been reserved. Let be $D(j)$ be the set of downlinks involved by VPCj. By updating $\Delta$ in the following manner: $\Delta^{\prime}=\Delta \backslash D(j)$, if another uplink time-slot is available to the GES, the above mentioned procedure can be repeated.

In conclusion, the 'stolen slot' procedure allows the GES to transmit $m$ cells - belonging to one connection each - towards $n$ downlinks $(n \geq m)$, assuming the following relations:

i) $\quad D(j) \subseteq \Delta, \quad j=1, \ldots, m$

ii) $\quad D(j) \cap D(i)=\varnothing, \quad \forall i \neq j$

iii) $n=\sum_{j=1}^{m} \operatorname{Card}\{D(j)\} \leq \operatorname{Card}\{\Delta\}$

For instance, Fig. 6 a), referred to the example of Fig. 8 , shows the sets $\Delta, D(1)$, $D(2)$ and $D(3)$, and shows that VPC1, 2 and 3 are eligible to steal the VPC0 time-slot since $D(j) \subseteq \Delta, j=1,2,3$. Assuming that the VPC1 and 2 have higher priority with respect to VPC3, the time-slot is stolen by VPC1, since $\operatorname{Card}\{D(1)\}>\operatorname{Card}\{D(2)\}$. Then, assuming that the GES has another available uplink time-slots, $\Delta$ is updated as shown in Fig. 6 b); by repeating the procedure, given that VPC2 and 3 are eligible, the time-slot is assigned to VPC2, which has higher priority with respect to VPC3. Finally, even if the GES has another free time-slot, it cannot be used by any VPC because $D(1), D(2)$ and $D(3)$ are not sub-sets of the updated $\Delta$, as shown in Fig. 6 c).

a)

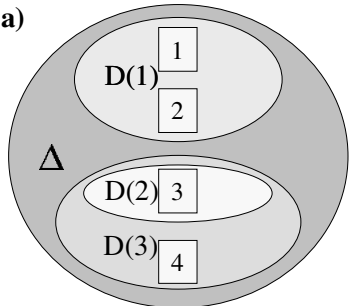

$\Delta=\{1,2,3,4\}$

$\mathrm{D}(1)=\{1,2\}$

$\mathrm{D}(2)=\{3\}$

$\mathrm{D}(3)=\{3,4\}$

Time-slot assigned to VPC1 b)

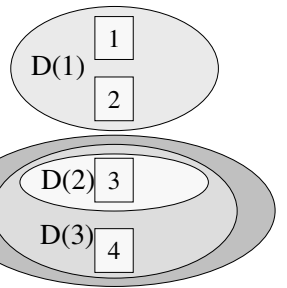

$\Delta^{\prime}=\Delta \backslash D(1)=\{3,4\}$

$\mathrm{D}(2)=\{3\}$

$\mathrm{D}(3)=\{3,4\}$

Time-slot assigned to VPC2 c)

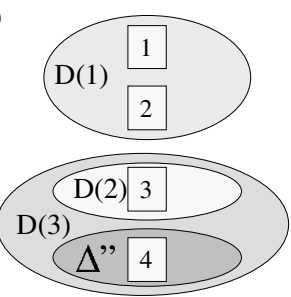

$\Delta^{\prime \prime}=\Delta^{\prime} \backslash \mathrm{D}(2)=\{4\}$

$D(3)=\{3,4\}$

Time-slot not assigned

Fig. 6. Downlink sets for the stolen slot procedure 


\section{OPNET Simulations}

In order to validate the proposed architecture, simulations have been performed with the OPNET tool by MIL3, which is a discrete event simulator specifically developed for simulating telecommunication networks.

The simulation scenario consists of a single gateway transmitting 8 one-to-one VCCs towards the satellite via a TDM uplink. In order to evaluate the performances of the proposed architecture, it is compared to the reference one, in which the VCCs are mapped onto the uplink frame after the connection set-up. The simulation parameters are summarised in Table 1.

Table 1. Simulation parameters

\begin{tabular}{|c|c|c|c|c|}
\hline $\begin{array}{c}\text { Uplink Capac- } \\
\text { ity }\end{array}$ & $\begin{array}{c}\text { N. of Time- } \\
\text { Slots per Frame }\end{array}$ & Frame Length & $\begin{array}{c}\text { Number of RT } \\
\text { Connections }\end{array}$ & $\begin{array}{c}\text { Number of RT } \\
\text { QoS Classes }\end{array}$ \\
\hline $9,088,000 \mathrm{bps}$ & 1136 & $53 \mathrm{~ms}$ & 8 & 3 \\
\hline
\end{tabular}

\begin{tabular}{|c|c|c|c|}
\hline QoS Class & PCR Range [bps] & CDV Range $[\mathbf{m s}]$ & Application Example \\
\hline 1 & $16,000-1,400,000$ & $10-100$ & Voice \\
\hline 2 & $64,000-40,000,000$ & $100-600$ & Video \\
\hline 3 & $9,600,00-1,500,000$ & $>600$ & Files \\
\hline
\end{tabular}

\begin{tabular}{|c|c|c|c|c|}
\hline VCC & PCR [bps] & CDV [ms] & QoS Class & $\begin{array}{c}\text { N. of Required Time- } \\
\text { Slots per Frame }\end{array}$ \\
\hline 0 & 600,000 & 15 & 1 & 75 \\
\hline 1 & $1,300,000$ & 1000 & 3 & 162.5 \\
\hline 2 & 64,900 & 600 & 2 & 8.1125 \\
\hline 3 & 10,000 & 1000 & 3 & 1.25 \\
\hline 4 & $1,400,000$ & 100 & 1 & 175 \\
\hline 5 & 31,000 & 10 & 1 & 3.875 \\
\hline 6 & $5,000,000$ & 1000 & 3 & 625 \\
\hline 7 & 682,100 & 600 & 2 & 85.2625 \\
\hline
\end{tabular}

Four simulation runs have been executed, as shown in Table 2.

Table 2. Simulation runs

\begin{tabular}{|c|c|c|c|}
\hline $\begin{array}{c}\text { Simulation } \\
\text { Run }\end{array}$ & Scenario & Source Rate & VCC Admission Order \\
\hline 1 & Reference & Nominal & VCC $0,1,2,3,4,5,6$ \\
\hline 2 & Reference & Nominal & VCC $0,1,2,3,4,6,5$ \\
\hline 3 & Proposed & Nominal & Not significant \\
\hline 4 & Proposed & Variable & Not significant \\
\hline
\end{tabular}

Note that, in the reference scenario, the last VCC that has to be mapped is VCC7; since the other VCCs requires a total amount of 1053 time-slots in order to be mapped onto the uplink frame (as a matter of fact, each VCC is mapped onto an integer num-

${ }^{1}$ The sources can transmit constantly at the PCR - nominal rate - or with a variable rate; the rate variations have a Gaussian distribution with the mean equal to PCR (however, the UPC blocks limit the buffer input rate to the PCR). 
ber of time-slots, e.g., VCC1 is mapped onto 163 time-slots), the leftover 83 time-slots are not sufficient to map VCC7. On the contrary, the proposed architecture is capable of accepting the last VCC, since it is not dependent on the frame structure; the sum of the PCRs of the VCCs is equal to the uplink capacity.

Fig. 7 shows the simulation results.
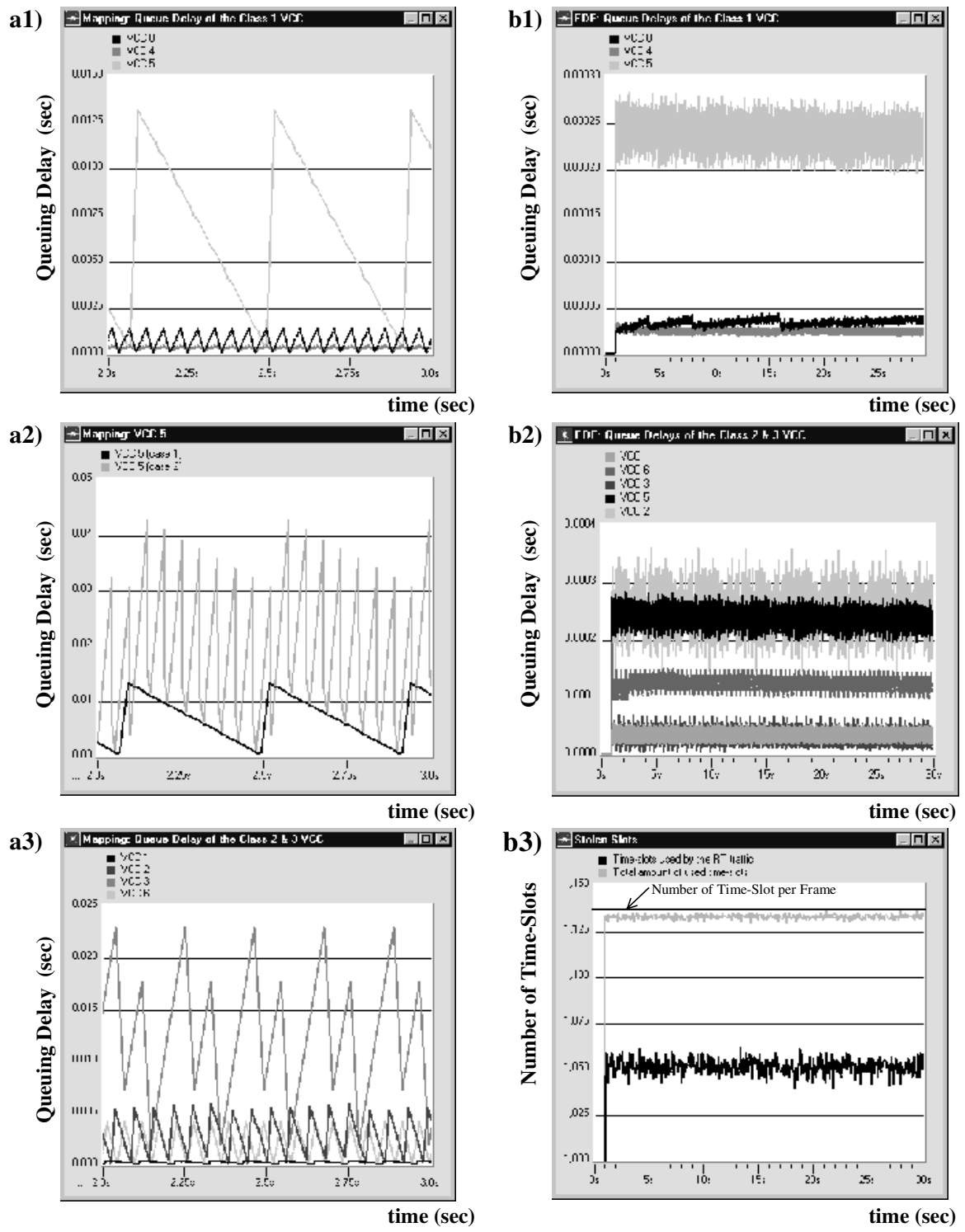

Fig. 7. Simulation results 
Fig. 7 a1) shows the queuing delay perceived by the packets of the Class 1 VCCs in Simulation 1. As explained in Section 2, assuming that the time-slots are regularly paced, the queuing delay depends on the PCR of the connection and not on the requested CDV. For instance, the light gray plot shows the queuing delay of VCC5, whose PCR is equal to 31,000 bps; thus, the expected maximum CDV is given by $T_{F R A M E} / N_{P C R}=53 \mathrm{~ms} / 4=13.25 \mathrm{~ms}$. The simulation confirms this prediction, and shows that the reference architecture cannot guarantee the requested CDV to VCC5. The periodic saw-tooth behaviour of the queuing delays depend on the fact that the sources are not synchronized with the periodicity of the assigned time-slots.

Fig. 7 a2) shows the queuing delay perceived by the packets of the Class 2 and 3 VCCs in Simulation 1. Also in this case, the queuing delays depend only on the PCR of the connections.

Fig. 7 a3) shows the comparison between the queuing delays perceived by the packets of VCC5 in Simulation 1 and 2. Since in Simulation 2 VCC5 is the last admitted connection, it has to be mapped onto an already heavily loaded uplink frame; thus, the time-slot allocation cannot be as regular as in the Simulation 1, and the maximum distance between two consecutive time-slots is greater. Thus, as shown in Fig. 7 a3), the CDV in Simulation 2 is even greater than the one in Simulation 1 (which was already unacceptable).

Fig. 7 b1) and b2) shows the queuing delay perceived by the packets of the VCCs in Simulation 3. In this case, the queuing delays are independent of the PCR and are much below the requested CDVs, even if the rate of the traffic entering the GES is equal to the uplink capacity - in Simulation 3, the gateway is supporting all of the 8 VCCs transmitting at full rate.

Finally, Fig. 7 b3) shows stolen slot algorithm performances. In Simulation 4, in which the sources transmit with variable rates, the GES transmits a NRT packet every time the stolen slot algorithm communicates that a time-slot is available (see Section 4). The lower plot represents the total transmission rate of the RT VCCs of Simulation 4, while the higher plot represents the total amount of transmitted packets, given by the RT traffic plus the NRT packets transmitted on the stolen slots; the simulation results show that the stolen slot algorithm allows the utilisation of more than $99.8 \%$ of the link capacity (note that, in the simulation, a NRT time-slot is available every time a time-slot is stolen).

\section{Conclusions}

In the present paper, a gateway architecture suitable for DVB-RCS satellite networks has been proposed. The architecture aims at supporting the RT priority traffic in the most efficient manner, without affecting the NRT traffic. At the same time, the scalability issue, which is relevant for the gateways because of the large number of supported traffic flows, is taken into account.

The three objectives are met:

i) The proposed scheduling scheme transmits the RT packets with strict priority with respect to the NRT traffic; the NRT traffic is protected by misbehaving RT sources 
(i.e., sources transmitting with a higher rate with respect to the contracted one) by the UPC functional blocks of the gateway, which have shaping and policing functionalities.

ii) Thanks to the 'stolen slot' concept, by exploiting the UPC blocks, the proposed 'stolen slot' algorithm is capable of re-distributing the unused capacity which was assigned to RT traffic among the NRT flows.

iii) The proposed two-levels aggregation policy, based on the definition of QoS classes within the RT priority traffic, reduces effectively the number of buffer the gateway has to manage.

Furthermore, the paper defines a procedure that allows the distribution of the available time-slots among unicast and multicast traffic flows.

Finally, simulations have been performed with the OPNET tool, which verified the effectiveness of the proposed buffering and scheduling schemes and of the proposed 'stolen-slot' algorithm.

Acknowledgements. The authors wish to thank Dr. Benoit Garnier of Alcatel Space Industries $(\mathrm{F})$ for his valuable contribution.

\section{References}

[1] Pietrabissa, S. Fiorido, "Access Layer Protocols for the GEOCAST Project", IST Mobile Communication Summit 2001, Sitges, September 2001

[2] European Broadcasting Union: "Digital Video Broadcasting (DVB): Interaction channel for satellite distribution systems", ETSI EN 301790 V1.2.2, http://www.etsi.org, December 2000

[3] European Broadcasting Union: "Digital Video Broadcasting (DVB);Interaction channel for Satellite Distribution Systems; Guidelines for the use of EN 301 790", ETSI TR 101790 V1.1.1, http://www.etsi.org, September 2001

[4] Garnier, "Access Layer Specification for GEOCAST System", GEOCAST Draft, October 2001

[5] ATM Forum Technical Committee: "Traffic Management Specification Version 4.1", www.atmforum.org, March 1999

[6] F. Delli Priscoli, A. Faggiano, V. Verrillo: "Uplink Access Technique in an ATM-based Satellite Network", Proc. of the 4th ACTS Mobile Communication Summit '99, Sorrento (Italy), June 1999.

[7] H. Koraitim, S. Tohme, H. Cakil, "MB-ICBT protocol performance in star-configured VSAT satellite networks", 2nd IEEE Symposium on Computers and Communications (ISCC '97), July 1997

[8] T. Örs, Z. Sun and B.G. Evans, "A MAC Protocol for ATM over Satellite", Proceedings of Sixth IEE Conference on Telecommunications, pp. 185-190, Edinburgh-UK, 29 March-1 April 1998

[9] Hung, M.-J. Monpetit, and G. Kesidis, "ATM via satellite: a framework and implementation”, ACM/ Baltzer WINET, 4(2):141-153, February 1998

[10] V. Firoiu, M. Borden:”A Study of Active Queue Management for Congestion Control”, IEEE INFOCOM '99

[11] Chengzhi Li and Edward W. Knightly, "Schedulability Criterion and Performance Analysis of Coordinated Schedulers", in Proceedings of ITC-17, September 2001 